\title{
Multiscale model of the human cardiovascular system: description of heart failure and comparison of contractility indices
}

\author{
S. Kosta, ${ }^{\text {a }}$, J. Negroni, ${ }^{\text {b }}$, E. Lascano, ${ }^{\text {b }}$, and P. C. Dauby ${ }^{\mathrm{a}}$ \\ ${ }^{a}$ GIGA - In Silico Medicine, University of Liege, Belgium (e-mail: sarah.kosta@ulg.ac.be). \\ ${ }^{b}$ Department of Comparative Cellular and Molecular Biology, Favaloro University, Buenos Aires, Argentina
}

\begin{abstract}
A multiscale model of the cardiovascular system is presented. Hemodynamics is described by a lumped parameter model, while heart contraction is described at the cellular scale. An electrophysiological model and a mechanical model were coupled and adjusted so that the pressure and volume of both ventricles are linked to the force and length of a halfsarcomere. Particular attention was paid to the extreme values of the sarcomere length, which must keep physiological values. This model is able to reproduce healthy behavior, preload variations experiments, and ventricular failure. It also allows to compare the relevance of standard cardiac contractility indices. This study shows that the theoretical gold standard for assessing cardiac contractility, namely the end-systolic elastance, is actually load-dependent and therefore not a reliable index of cardiac contractility.
\end{abstract}

Keywords: Mathematical models - Physiological models - Multiscale models - Cardiovascular system - Sarcomere contraction

\section{Introduction}

Mathematical models of biological systems have become a powerful tool for cardiovascular sciences. These models allow for a variety of studies that are generally difficult to implement experimentally.

A complete model of the whole human cardiovascular system (CVS) requires a mathematical description of two components:

- The cardiac pump, composed of two atria and two ventricles;

- The vascular network (veins, arteries, capillaries, ...).

The heart contraction is often described with ad hoc models, like the time-varying elastance model $[1,2,3]$. Such macroscopic models are not based on the cardiac tissue

\footnotetext{
Email address: sarah.kosta@ulg.ac.be (S. Kosta,
}

properties and cannot reproduce behaviors that arise from the microscopic scale. In this work, a cardiac cell contraction model is used and integrated at the organ level in order to get a multiscale model of the human CVS [4, 5]. The purpose of this model is to link macroscopic properties to the microscopic behaviors they originate from, a correlation impossible to establish with phenomenological models.

\section{Methods}

There is always a balance to be found between a sophisticated model and computational efficiency. When modeling complex biological systems like the CVS, assumptions and simplifications have to be made in order to get a reasonable computational time. As far as our CVS model is concerned, we wanted a short computational time in order to study physiological behaviors at the whole CVS scale. In 
this section we describe our CVS model and the assumptions we had to make in order to get a computationally efficient model.

\subsection{The vascular network}

Blood travels unidirectionally across the body through blood vessels. Leaving the left atrium and ventricle, it flows sucessively through systemic arteries, capillaries, veins and goes back to the right atrium and ventricle. It is then sent through pulmonary arteries, capillaries and veins. It eventually goes back to the left atrium and ventricle and the cycle starts all over again, as depicted in Fig. 1. In this work, we assimilate this complex system composed of many different vessels to a 6-chamber model. Four chambers are assimilated to elastic "balloons" that can be filled with blood and the other two represent the cardiac pump. The fluid mechanics equations that govern such a system are described elsewhere $[2,5,6]$ and only the cardiac pump model will be described in detail in the next section.

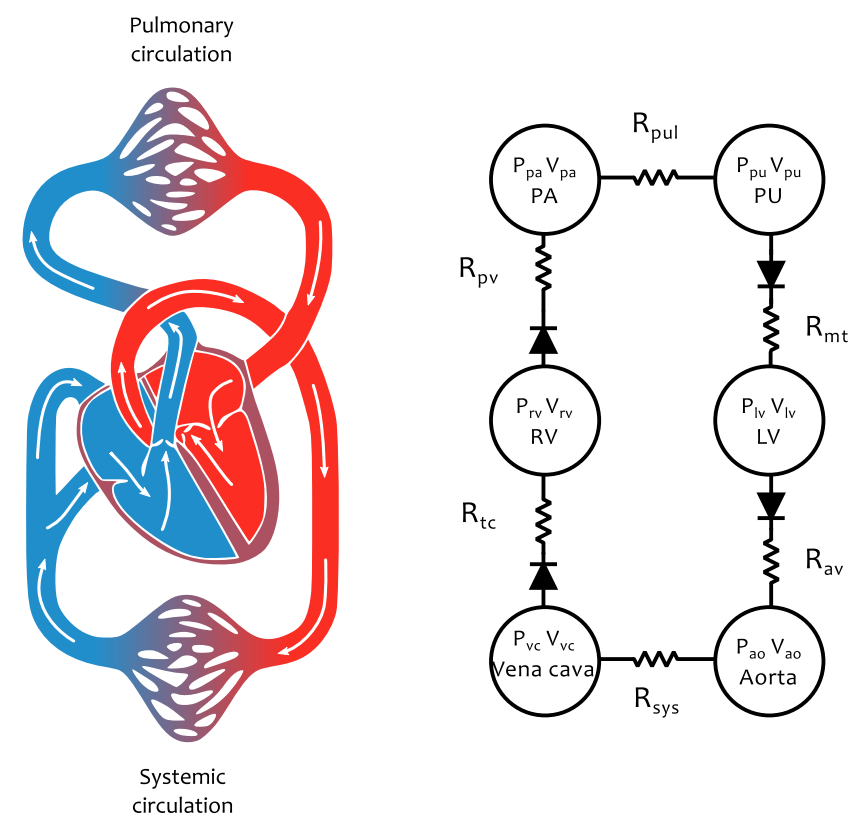

Figure 1: Left: Representation of the cardiovascular system. Right: Diagram of the 6-chamber hemodynamic model. Left ventricle (LV), right ventricle $(\mathrm{RV})$, pulmonary artery $(\mathrm{PA})$, pulmonary vein $(\mathrm{PU})$, aorta $(\mathrm{AO})$, vena cava (VC).

\subsection{The cardiac pump}

The cardiac pump is composed of two atria and two ventricles. Here we only model the ventricles, as they hold the major role in ejecting the blood through the systemic and pulmonary circulations. Thus we described the cardiac pump with only two chambers, the left and right ventricles.

The major difference with the other four chambers of the CVS model is that ventricles are able to actively contract and generate pressure. Therefore a passive pressurevolume relationship of the form $P(t)=E \cdot V(t)$ (where $E$ is the constant elastance of the chamber) is not suitable in this case. A convenient solution would be to use a similar equation, but with a time-dependent elastance. The time dependence would then be fitted to experimental data in order to get physiological results. This ad hoc approach (called the time-varying elastance model) has been extensively used to model cardiac contraction [1, 2, 3]. It has the advantage of providing a very simple mathematical description of active contraction and can lead to consistent results. However this model has some limitations. It is based on the assumptions that the end-systolic pressurevolume relationship (ESPVR) is linear and unique, even though experiments have shown this curve to be more parabolic than linear $[7,8]$ and load-dependent [9]. Furthemore, the ventricular pressure has been shown to be dependent on the flow out of the ventricle $[10,11]$. Subsequent modifications to this model have been proposed to account for the non linear ESPVR and the flow-dependent pressure $[10,11,12]$. However, these ad hoc modifications can not overcome the main drawback of this model, namely the absence of connection with the physiology of cardiac contraction.

We choose a more physiological approach and described cardiac contraction at the cellular scale instead $[4,5,13]$. This heart model is described in the following sections. 


\subsubsection{Cell model}

Cardiac cells are excitable and contractile. When an action potential (AP) arises, the cell is able to contract through the excitation-contraction process. We followed the approach of Puglisi et al. [14] to build a human cardiac cell model: we connected an electrophysiological model of a human ventriclar cell [15] to a mechanical contraction model of a half-sarcomere $[16,17,18]$. Those two models are described below.

\section{Electrophysiology}

An electrophysiological model of an excitable cell is able to reproduce the AP across the cell membrane, i.e. the time evolution of the membrane electric potential $V$. This potential varies because massive quantities of ions cross the membrane (leading to ionic currents) during an AP. The equation governing the time evolution of $V$ is given by:

$$
C_{m} \frac{\mathrm{d} V}{\mathrm{dt}}+\sum_{j} I_{j}+I_{s t i m}=0
$$

where $C_{m}$ is the membrane capacitance, $I_{j}$ is the electrical current carying ion $j$ and $I_{\text {stim }}$ is a stimulation current that triggers the AP.

From the ionic currents we can also obtain the time evolution of the intracellular concentrations for each type of ions:

$$
\frac{\mathrm{d}[\mathrm{Ion}]_{i}}{\mathrm{dt}}=\frac{I_{\text {in }}-I_{\text {out }}}{z_{\text {ion }} V_{c} F}
$$

where $I_{\text {in }}$ (resp $I_{\text {out }}$ ) is the global electrical current carrying the ions inside (resp. outside) the intracellular compartment of volume $V_{c}, z_{i o n}$ is the valence of the ion, and $F$ is the Faraday constant.

An appropriate description of the ionic currents is required to obtain physiological results. More information can be found in the original paper [15] regarding the mathematical expressions of all the ionic currents.

\section{Mechanical contraction}

Cardiac cells contain basic contractile units called sarcomeres, schematized in Fig. 2. A sarcomere is mainly composed of actin (thin) and myosin (thick) filaments. In presence of calcium and ATP, a myosin head (also called a crossbridge, noted $\mathrm{CB}$ ) is able to attach to an actin molecule and rotate its head, thus pulling the actin filament. The active force produced by a sarcomere is related to the force produced by the pulling (also called the power stroke) of the myosin head. There is also a passive contribution to the total produced force because of the sarcomere elastic properties.
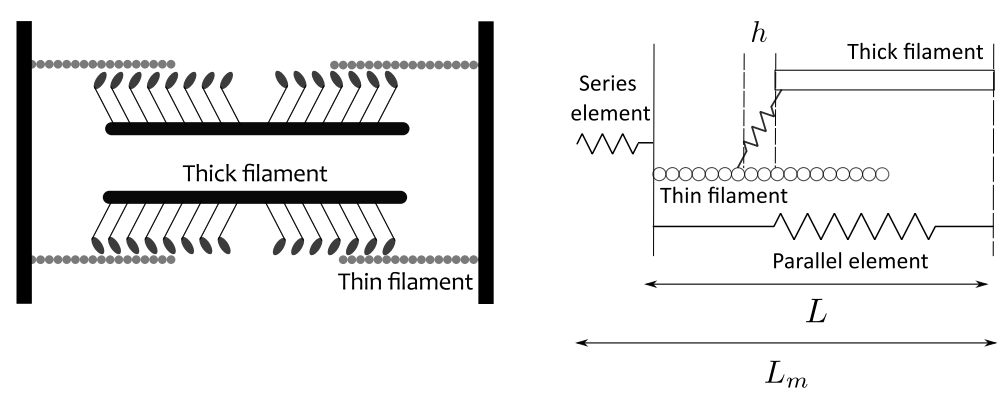

Figure 2: Left: Representation of the thin and thick filaments of a sarcomere. Right: Mechanical model of a half sarcomere (adapted from [16]).

We use the model of Negroni and Lascano $[16,17,18]$ to describe the contraction of a half-sarcomere, composed of a half-thick and a half-thin filaments (see Fig. 2). Only a brief summary of the model is given below, but a more detailed explanation can be found in the orginal papers. This model focuses on the behavior of an equivalent crossbridge that representents all the crossbridges of the half-thick filament. It is assimilated to a linear spring of horizontal elongation $h$ that is always attached to the half-thin filament (otherwise the force would suddenly go to zero, which is not physiological).

The active force is proportional to the spring elongation $h$ but also to the concentrations of attached myosin heads. These concentrations can be determined from the intracellular calcium kinetics depicted in Fig. 3. Calcium kinetics is described with a 5-state model (two states from 
the original paper [17] were merged into one [18]). A troponin system (TS) is composed of three adjacent troponintropomyosin units and can fix three calcium ions in one step $\left(\mathrm{TSCa}_{3}\right)$. Then three crossbridges can attach to the actin molecules in a weak pre-power stroke state $\left(\mathrm{TSCa}_{3}^{\sim}\right)$, followed by a state where they are able to develop a power stroke $\left(\mathrm{TSCa}_{3}^{*}\right)$. Finally the three calcium ions can detach (TS*) and the crossbridges eventually detach to end the cycle. The active force is proportionnal to the concentrations of the three states with attached crossbridges $\left(\left[\mathrm{TSCa}_{3}^{\sim}\right],\left[\mathrm{TSCa}_{3}^{*}\right]\right.$, and $\left.\left[\mathrm{TS}^{*}\right]\right)$.

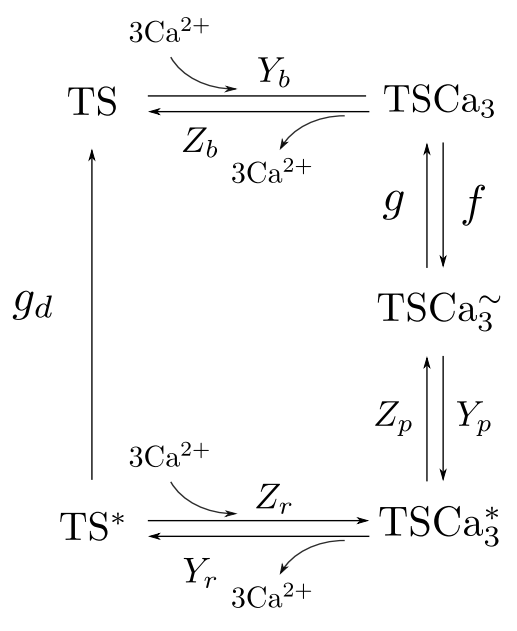

Figure 3: Calcium kinetics. Adapted from [17].

There is a strong relationship between the force and length of a cardiac cell. This leads to important properties at the organ scale, especially the Frank-Starling mechanism (the greater the ventricular filling, the greater the pressure). Therefore it is worth noticing that some rate constants in the calcium kinetics from Fig. 3 depend on the length $L$ of the half-sarcomere, leading to a length-dependent force. The passive elastic properties of the half sarcomere are modeled with a parallel elastic element shown in Fig. 2. Eventually, a series elastic element is also added to the half sarcomere in order to account for the elastic ends of a muscle fiber, as shown in Fig. 2. Thus the total length of the half sarcomere is noted $L_{m}$ and we have:

$$
L_{m}=L+L_{s}
$$

where $L_{s}$ is the length of the series elastic element.

\section{Whole cell model}

We connect the electrophysiology and mechanics as already done by Puglisi et al. [14]. The intracellular calcium concentration $\left(\left[\mathrm{Ca}^{2+}\right]_{\mathrm{i}}\right)$ obtained from the electrophysiological model is used as an input for the calcium kinetics. We also make some small modifications regarding the electrophysiological model [15] and the mechanical model [18]. These modifications are described below.

- We add a feedback pathway from mechanics onto electrophysiology. To this end, an additional current is added in equation (1) for $\left[\mathrm{Ca}^{2+}\right]_{\mathrm{i}}$. This current is the troponin current and takes into account the buffering of three calcium ions by the troponin systems defined previously. It is expressed as follows:

$$
I_{\text {trop }}=3\left(\frac{\mathrm{d}\left[\mathrm{TSCa}_{3}\right]}{\mathrm{dt}}+\frac{\mathrm{d}\left[\mathrm{TSCa}_{3}^{\sim}\right]}{\mathrm{dt}}+\frac{\mathrm{d}\left[\mathrm{TSCa}_{3}^{*}\right]}{\mathrm{dt}}\right)
$$

We then have to reduce the total cytosolic calcium buffer concentration $\left(\mathrm{Buf}_{\mathrm{c}}\right.$ in the original paper) from 200 to $130 \mu \mathrm{M}$ since the buffering due to troponin in the cytoplasmic compartment is now taken into account in equation (1).

- The sliding velocity of the equivalent crossbridge along the thin filament has to be increased by a factor of 3.5. Otherwise, the produced force is not able to increase fast enough compared to $\left[\mathrm{Ca}^{2+}\right]_{\mathrm{i}}$ increase during an AP. It should be stated that, unlike the rate constants describing calcium kinetics, the parameter governing the sliding dynamics of the equivalent crossbridge can not be unequivocally connected to the underlying molecular mechanisms of filament sliding. Several values have been proposed $[4,16,17$, 18] and, in our case, increase in sliding velocity can 
be seen as the price to pay in order to get physiological values for the developed force and the resulting ventricular pressure. Note that the adjusted value remains close (in term of order of magnitude) to the initial value from [18].

- The $\left[\mathrm{Ca}^{2+}\right]_{\mathrm{i}}$ increase obtained with the ten Tusscher and Panfilov model [15] is rather sharp and leads to a slight increase of the elongation $h$ (from the mechanical model) at the beginning of sarcomere contraction. This behavior does not impact significantly the global hemodynamic results, but a smoother increase of $\left[\mathrm{Ca}^{2+}\right]_{\mathrm{i}}$ would ensure a more physiological behavior of the equivalent crossbridge elongation $h$. To this end, we follow the approach of Lascano et al. [19] and adapt the equations governing calcium liberation by the sarcoplasmic reticulum. In our previous published paper [6], we were still using the original electrophysiological model of ten Tusscher and Panfilov model.

- The parameters characterizing the passive force in the mechanical model have to be adapted. A more detailed explanation is provided at the end of section 2.2 .3 .

The complete set of equations and parameters used in the cell model can be found in the Supplementary Material [20].

\subsubsection{Ventricular model}

At the ventricular scale, the action potential propagation across cardiac tissues leads to a global contraction of the ventricle. Thus, there is a link between the force produced by the cardiac cells and the global pressure inside ventricular cavities. Likewise, length variations of cardiac cells are related to volume variations of ventricular cavity. Connecting microscopic variables (force and length) to macroscopic variables (pressure and volume) results in a multiscale model of heart contraction. To achieve this, we followed an approach similar of that of Shim et al. [4]. To connect the force $F_{m}$ and total length $L_{m}$ of the halfsarcomere to the pressure and volume of the active chamber, both ventricles are simply considered as spheres, as shown in Fig. 4.
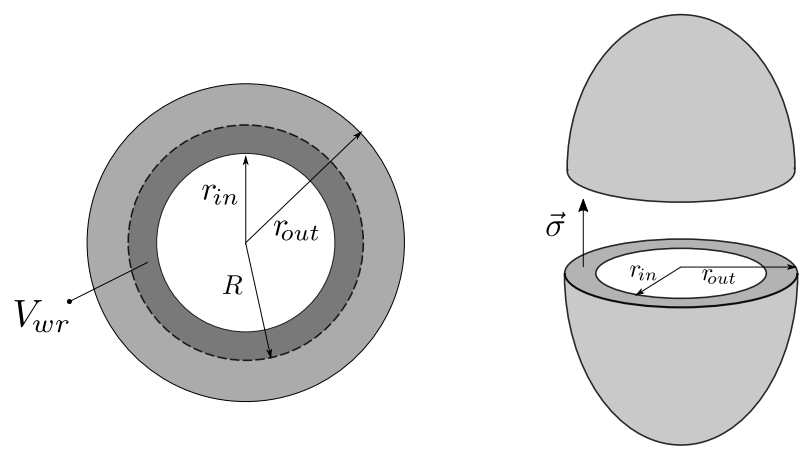

Figure 4: Spherical ventricle model. Left: Top view of the ventricle. $N$ sarcomeres are aligned along a circle of radius $R$ (dotted black). Right: Both ventricles are assimilated to thin spheres. The wall stress $\sigma$ is related to the force produced by the sarcomeres and allows for the calculation of the pressure inside the ventricle.

As already done by Shim et al. [4], we assume that $N$ half-sarcomeres of total rest length $L_{m 0}$ are aligned along a circle of radius $R_{0}$ :

$$
L_{m 0}=\frac{2 \pi R_{0}}{N} .
$$

This radius was chosen so that the total half-sarcomere length $L_{m}$ during a healthy heartbeat varies between physiologically relevant extremes, i.e. between 0.98 and 1.115 $\mu m[21]$. As explained earlier, length-dependent properties at the cellular scale are connected to macroscopic properties of the heart. Thus it was essential to get the correct interval of variation for $L_{m}$ in our multiscale model. It is worth noticing that the model of Shim et al. [4] did not include the series elastic element from [18] and that the length variations did not belong to the physiological interval defined here.

The blood volume inside the ventricle is given by:

$$
V_{i n t}=\frac{4}{3} \pi r_{i n}^{3}
$$


This volume varies in time during a heartbeat. Thus $L_{m}$ is given by:

$$
L_{m}=\frac{2 \pi R}{N}
$$

where $R$ is calculated using:

$$
V_{i n t}+V_{w r}=\frac{4}{3} \pi R^{3}
$$

In this relation, $V_{w r}$ is the constant volume of the incompressible wall included between $r_{\text {int }}$ (at the beginning of ventricular filling) and $R$.

Now we have to connect the pressure $P$ inside the ventricular cavity to the force $F$ produced by the half-sarcomeres. We assume that many contractile units are distributed homogeneously in all directions on the ventricular wall [4], so that the wall stress $\sigma$ can be considered uniform. With a constant wall stress $\sigma$, the equilibrium of the two hemispheres of the ventricles gives the following expression of the pressure inside the active chambers:

$$
P=\sigma\left(\frac{r_{\text {out }}^{2}}{r_{\text {in }}^{2}}-1\right)
$$

where $\sigma$ is given by:

$$
\sigma=\frac{F}{A}
$$

with $F$ the force and $A$ the cross-sectional area.

The mechanical contraction model actually provides $F_{m}$, the force normalized with respect to the muscle crosssectional area measured at a defined reference state, $A_{r}$ :

$$
F_{m}=\frac{F}{A_{r}} .
$$

Assuming that muscle units keep constant volume, we also have:

$$
L_{r} \cdot A_{r}=L_{m} \cdot A
$$

where $L_{r}$ is the total sarcomere length at the reference area $A_{r}$.
From equations (8) - (10), we can obtain the pressureforce relation of our multiscale model :

$$
P=7.5 F_{m} \frac{L_{m}}{L_{r}}\left(\frac{r_{\text {out }}^{2}}{r_{\text {in }}^{2}}-1\right)+\lambda\left(V_{\text {in }}-V_{0}\right)^{3} .
$$

The 7.5 factor stands for the unit changes in equation (11) (pressure is expressed in $\mathrm{mmHg}$ while the normalized force is expressed in $\mathrm{mN} / \mathrm{mm}^{2}$ ) and the last term is a passive pressure that accounts globally for the elastic properties of the tissue surrounding the ventricle ( $\lambda$ is related to the stiffness and $V_{0}$ is the unstressed volume [22]).

\subsubsection{Heart model}

As explained before, we only include the ventricles in our heart model. To reduce the computationnal cost, both ventricles are assumed to have the same electrophysiology even if the mechanical behavior can be different. Thus the troponin currents described in equation (2) are averaged (and approximated) as follows:

$$
\begin{gathered}
I_{\text {trop }}=\frac{1}{2} 3\left(\frac{\mathrm{d}\left[\mathrm{TSCa}_{3}\right]}{\mathrm{dt}}+\frac{\mathrm{d}\left[\mathrm{TSCa}_{3}^{\sim}\right]}{\mathrm{dt}}+\frac{\mathrm{d}\left[\mathrm{TSCa}_{3}^{*}\right]}{\mathrm{dt}}\right)_{\mathrm{LV}} \\
+\frac{1}{2} 3\left(\frac{\mathrm{d}\left[\mathrm{TSCa}_{3}\right]}{\mathrm{dt}}+\frac{\mathrm{d}\left[\mathrm{TSCa}_{3}^{\sim}\right]}{\mathrm{dt}}+\frac{\mathrm{d}\left[\mathrm{TSCa}_{3}^{*}\right]}{\mathrm{dt}}\right)_{\mathrm{RV}}
\end{gathered}
$$

where the indices "LV" and "RV" refer respectively to the left and right ventricle.

\subsection{Parameter adjustment}

The multiscale model presented above depends on many parameters, which can be split into microscopic (or cellular) parameters and macroscopic (or hemodynamic) parameters.

The cellular parameters can be obtained in the Supplementary Material [20] or in the original papers in which the electrophysiological model and the contractile model were developed ( $\mathrm{M}$ cell in $[15],[17,18])$. As explained in the previous section, some parameter values were adapted (see Table A.1) and some equations were modified [19]. 
The nine hemodynamic parameters (resistances and elastances of passive chambers, wall thickness of the ventricles) were optimized using the fminsearch algorithm from MATLAB (The MathWorks, Natick, MA, USA) in order to minimize the absolute relative error between a chosen set of reference variables and their corresponding calculated values. This set of reference values included: the stroke volume, the left and right ventricular pressures, the minimal left and right ventricular volumes, the mean aortic and pulmonary artery pressures, and the amplitude of vena cava and pulmonary pressures. The values for these reference data were chosed in order to correspond to standard healthy values $[23,24]$. The valve resistances are not easy to adjust precisely but their values do not influence much the global hemodynamic results [25].

Note however that, with the set of cellular parameters described above, the model always produces high enddiastolic pressures and low end-systolic pressures compared to physiological values. This difficulty originates from the too simple geometrical description of the ventricles, which are here considered as spheres. In a real heart, bundles of cardiac fibers are actually wrapped around the ventricular cavity so that the ventricle is also twisted in a very complex way during contraction. Of course the spherical model of the ventricles presented above cannot take into account this aspect of contraction and an adaptation of the values of some parameters was necessary to compensate for the simplicity of the model.

From equation (7), it is easy to understand that systolic pressure, which is related to the maximum active force generated in the sarcomeres, can be increased by increasing the ventricular wall thickness. However, since the diastolic pressure, which is related to the passive force in the myocytes, also increases with the wall thickness, we had to reduce the intensity of the sarcomere passive force by $70 \%$ in order to recover physiological diastolic pressures. Note that Shim et al. [4] also modified the passive force expression in order to preserve the ventricular ejection fraction.
In summary, the price to pay for the spherical model of the ventricles is a reduction factor to apply to the cellular parameter measuring the passive force of the sarcomere. The adjusted parameters are given in the appendix A.1. Motivations for a better description of cardiac contraction at the cellular scale with a reasonable computational cost lead to the multiscale approach described in this section, which needed some phenomenological adjustments. Thus, this CVS model lies in the middle ground between pure phenomenological models, like the time-varying elastance, and more sophisticated 3D models for the ventricle [26, 27]. The former can not inherently account for cardiac properties that originates at the cellular scale while the latter proposes more realistic description of the ventricle but makes studies at the whole CVS scale arduous, partly because of the high computational cost.

\section{Results}

All simulations are performed in MATLAB (The MathWorks, Natick, MA, USA) using the stiff ordinary differential equations solver ode15s.

\subsection{Baseline}

With the set of parameters described in the previous section, we are able to obtain baseline results that correctly reproduce healthy behaviors of the CVS. Some important cellular variables are represented on the top panel of Fig. 5: the action potential, the intracellular calcium and the normalized force produced by the cell. The sarcomere length and ventricular volume for the left ventricle are also drawn, and it is worth noticing that the sarcomere length lies between physiological values [21]. The last panel shows the correponding pressure-volume loops (PV-loops). We also represented other hemodynamic variables in Fig. 6.

\subsection{Heart failure}

It has been shown that heart failure originates at the cellular scale $[28,29,30]$. Alterations in ionic currents and calcium handling lead to a prolonged action potential, a lower 

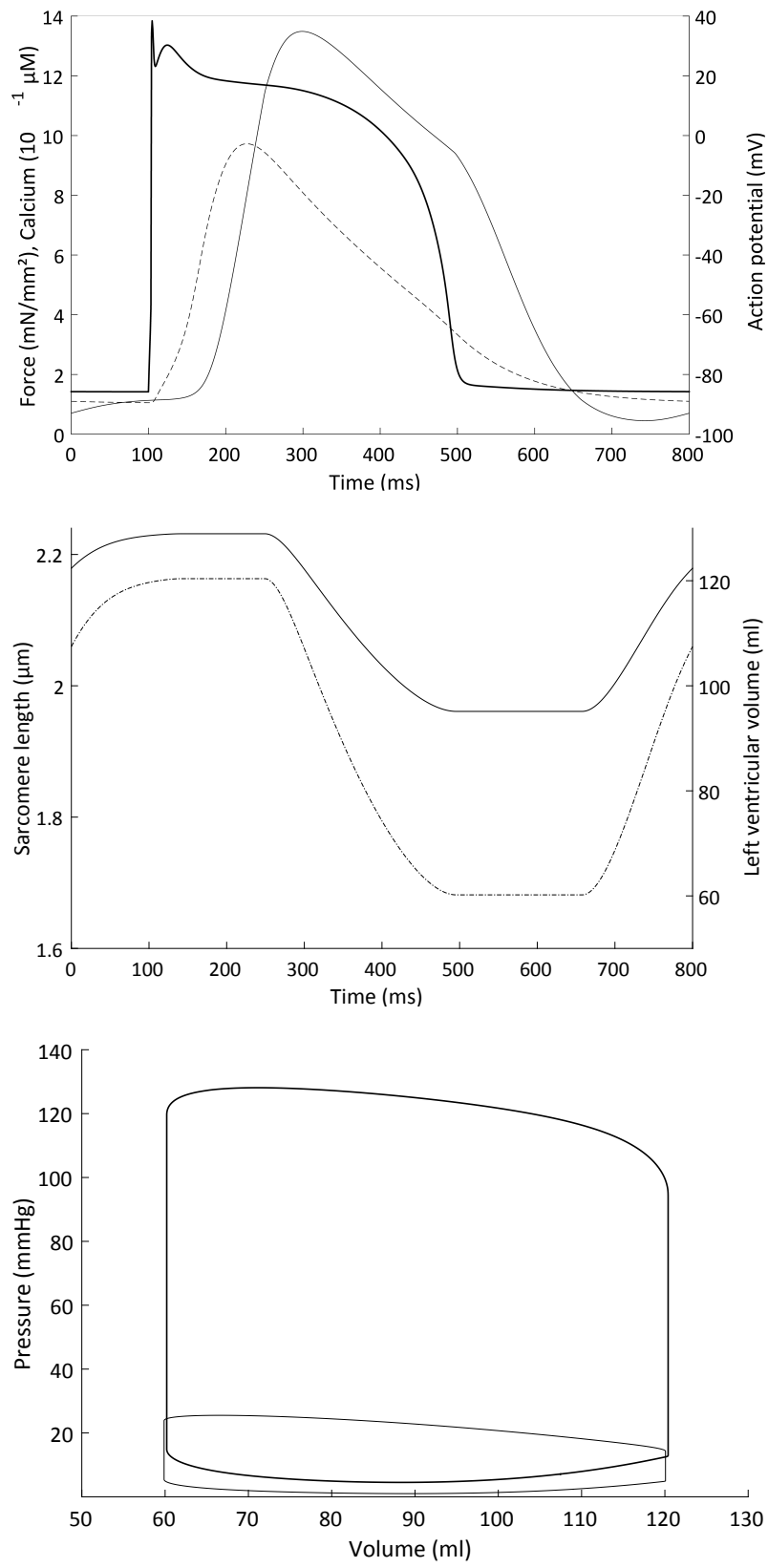

Figure 5: Top panel: Action potential (bold), normalized force (normal), and intracellular calcium (dashed) time evolution during one heartbeat in the left ventricle (heart rate $=75 \mathrm{bpm}$ ). Middle panel: Sarcomere length (normal) and left ventricular volume (dash-dotted) time evolution during the same heartbeat. Bottom panel: Pressurevolume loop in the left (bold) and right (normal) ventricle during the same heartbeat.

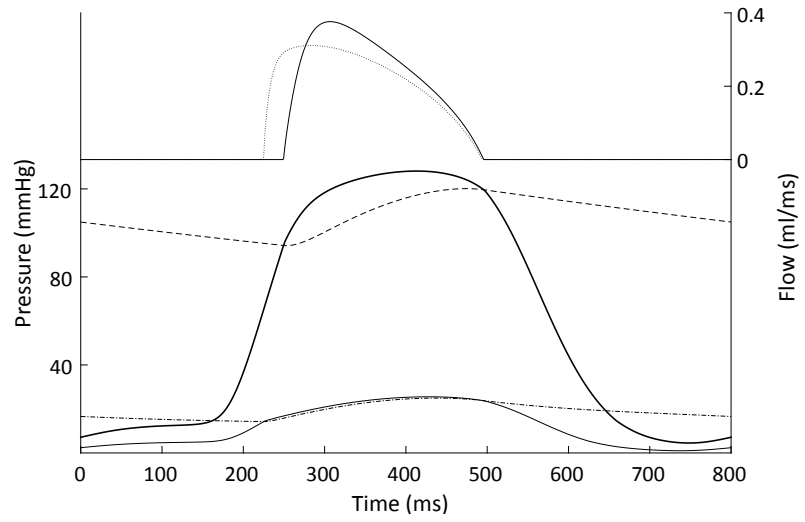

Figure 6: Hemodynamic variables. The top two curves represent the flow through the aortic valve (full line) and through the pulmonary valve (dotted) during one heartbeat. Left ventricular pressure (bold), aortic pressure (dashed), right ventricular pressure (normal) and pulmonary pressure (dash-dotted) are also represented.

peak of intracellular calcium, and a lower force. Reduction in current densities of the inward rectifier $\mathrm{K}^{+}$current and the transient outward $\mathrm{K}^{+}$current, increased activity of the $\mathrm{Na}^{+} / \mathrm{Ca}^{2+}$ exchanger and a reduced $\mathrm{Ca}^{2+}$ sequestration by the sarcoplasmic reticulum were implemented in our model $\left(G_{K 1}, G_{t o}\right.$ and $G_{K s}$ decreased by $20 \%, k_{N a C a}$ increased by $20 \%$ and $V_{u p}$ decreased by $10 \%$ with respect to their original values from [20]). Our multiscale model is then able to connect these cellular altered properties to the hemodynamics variables, as shown in Fig. 7.

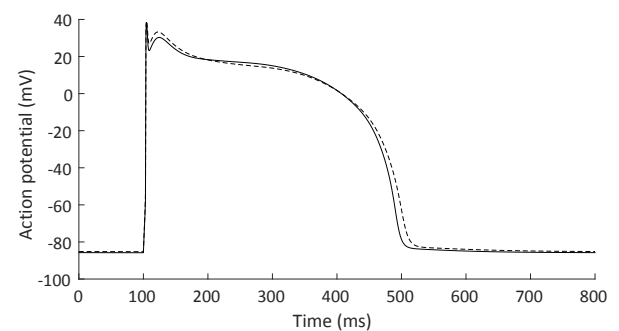

Figure 7: Ventricular failure (dashed) versus normal ventricle. From top to bottom and left to right: action potential, intracellular calcium, LV normalized force, LV and RV pressure-volume loops. 


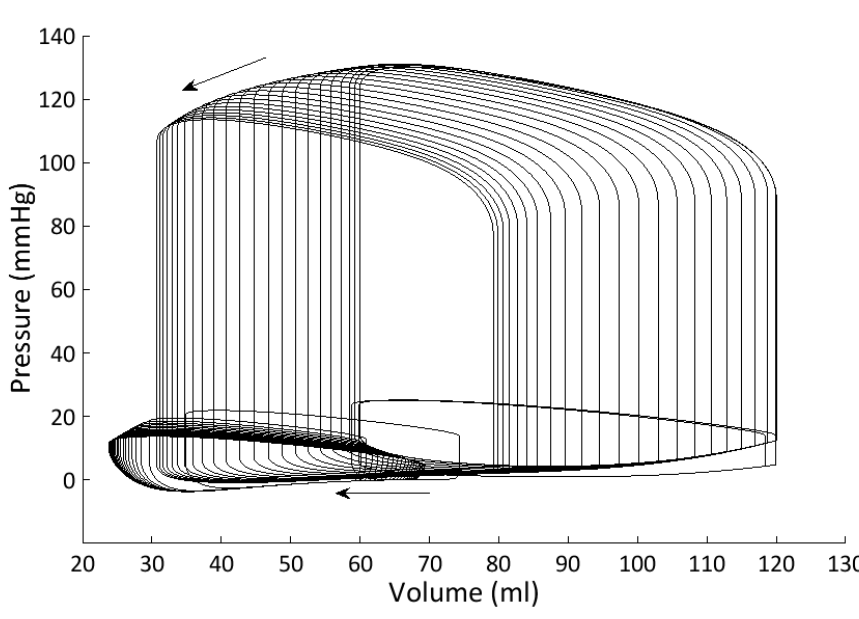

Figure 8: Decrease in preload in the right ventricle.

\subsection{Load variations}

Variations in preload or afterload during the cardiac cycle may happen for numerous reasons: a change in the venous return, a change in the arterial pressure, a pathological condition, etc. The inflation of a Fogarty balloon inserted in the vena cava is a current way of reducing preload experimentally. To mimic the inflation of this balloon, we increase the tricuspid valve resistance $\left(R_{t c}\right.$ from Fig. 1) tenfold, thus allowing less blood to enter the right ventricle. The consequences on the pressure-volume loops on both ventricles are presented in Fig. 8. It is worth emphasizing that the upper left corners of the PV-loops are not exactly located on a straight line [5], as often postulated when time-varying elastance models of contraction are used.

\subsection{Inotropy variations}

Changes in calcium handling at the cellular scale lead to changes in contraction at the organ scale. These changes are called inotropic variations. Inotropy is modulated by many cellular mechanisms, including calcium release and calcium uptake by the sarcoplasmic reticulum [31]. An increase (resp. decrease) in inotropy is modeled in our model with an increase (resp. decrease) in the rate of release $\left(V_{r e l}\right.$ from [20]) and uptake ( $V_{u p}$ from [20]) of calcium ions by the SR. Seven intracellular calcium curves are shown in Fig. 9. We have considered first the baseline situation described in Section 3.1 and we have then increased (resp. decreased) $V_{u p}$ and $V_{\text {rel }}$ by 5, 10, and $15 \%$. The corresponding left ventricular PV-loops are also shown in Fig. 9 .

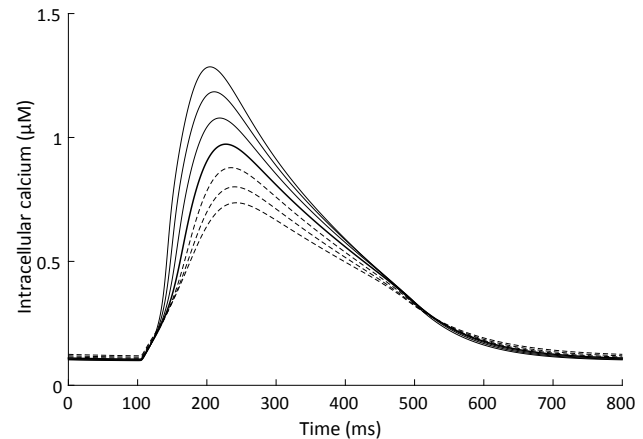

Figure 9: Left panel: intracellular calcium for seven different inotropic states: baseline (bold), inotropy increase (full line), inotropy decrease (dotted line). Right panel: corresponding left ventricular PV-loops.

\subsection{Cardiac contractility indices}

Assessing the left ventricular contractile (or inotropic) state of a patient is an ongoing preoccupation for clinicians. A good index of cardiac contractility should vary only with the inotropic state of the heart and be perfectly loadindependent. Many cardiac indices have been proposed to assess ventricular contractile state $[32,33,34]$ and here we compare some of these with our model.

The end-systolic pressure-volume relationship (ESPVR, see Fig. 10) is often considered as the best tool for assessing cardiac contractility because the slope of this curve, named the end-systolic elastance $\left(\mathrm{E}_{\mathrm{es}}\right)$, is assumed to be load-independent. However, the ESPVR has been shown to be load-dependent and non linear $[5,7,8,9]$. We also showed the non linearity of the ESPVR in Fig. 8 and its load-dependence will be observed below.

Another contractility index with a relatively weak afterloaddependence is the peak first derivative of left ventricular pressure, $(\mathrm{dP} / \mathrm{dt})_{\max }[32]$. In clinic, it is however easier to assess the arterial pressure than the ventricular pressure, 


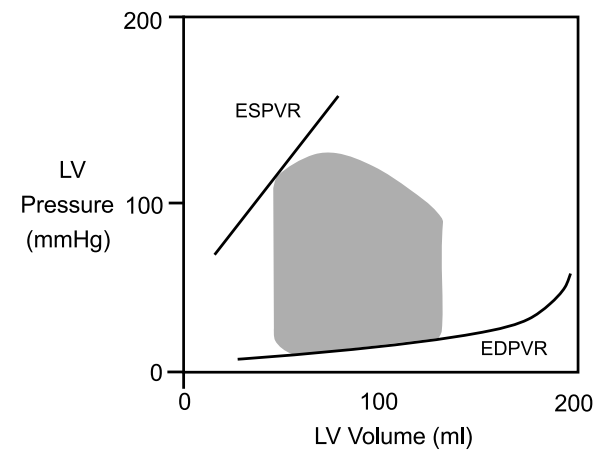

Figure 10: End-diastolic and end-systolic pressure-volume relationships. The slope of the ESPVR gives $\mathrm{E}_{\mathrm{es}}$. Adapted from [31].

so the arterial $(\mathrm{dP} / \mathrm{dt})_{\max }$ was also proposed as a cardiac contracility index [34].

In case of catheterization of the patient, the time $T_{d}$ from onset of contraction to left ventricular $(\mathrm{dP} / \mathrm{dt})_{\max }$ was proposed as a reliable contractility index [33].

To compare the different indices of contractility with our model, these were calculated for the seven states of inotropy described in the previous section (including the baseline case). In order to evaluate the possible loaddependency of the indices, we have induced six load variations by changing some hemodynamic parameters: $R_{\text {sys }}$ increased and decreased by $20 \%$, all the hemodynamic resistances increased and decreased by $10 \%$, all the elastances of the passive chambers increased and decreased by $10 \%$. For each load variation, $\left(\mathrm{dP}_{\mathrm{LV}} / \mathrm{dt}\right)_{\max },\left(\mathrm{dP}_{\mathrm{AO}} / \mathrm{dt}\right)_{\max }$ and $T_{d}$ were calculated on a single PV-loop after the system had reached a stabilized behavior. The results are shown in Fig. 11. The first two indices are increasing functions of inotropy while $T_{d}$ is a decreasing funtion of inotropy. With such load variations simulations, $\left(\mathrm{dP}_{\mathrm{LV}} / \mathrm{dt}\right)_{\max }$ standard deviation with respect to the baseline case is less than $1 \%$, while for $\left(\mathrm{dP}_{\mathrm{AO}} / \mathrm{dt}\right)_{\max }$ it ranges from 5 to $10 \%$ and for $T_{d}$ it ranges from 1 to $5 \%$. In the case of $\left(\mathrm{dP}_{\mathrm{LV}} / \mathrm{dt}\right)_{\max }$, the standard error is very small, which allows us to conclude that it can actually be considered as a reliable index of contractility, truly independent of the load and increasing with inotropy. The quan- tities $\left(\mathrm{dP}_{\mathrm{AO}} / \mathrm{dt}\right)_{\max }$ and $T_{d}$ have larger standard deviation, which indicates that those indices are slightly loaddependent.

The end-systolic elastance cannot be calculated with a single PV-loop provided that a linear regression between at least two end-diastolic points is required to calculate $E_{e s}$. Once the system had reach a stabilized behavior, a Fogarty protocol (described earlier) was induced in order to get more than one PV-loop. The ESPVR was calculated over four end-diastolic points after twenty PV-loops, as shown in Fig. 12. The calculated $E_{e s}$ is then represented as a function of inotropy. It is an increasing function of inotropy and the standard deviation ranges between $1 \%$ (low inotropy) and $94 \%$ (high inotropy). For this reason, the end-systolic elastance cannot be considered as a reliable index of contractility.

\section{Discussion}

We have developed a multiscale model of the human CVS based on the ten Tusscher and Panfilov model of electrophysiology [15], the Negroni and Lascano model of sarcomere contraction [18], and a lumped parameter model of hemodynamics.

The ventricular model is quite simple compared to other works [26, 27], but this approach offers a low computational cost. The aim of our work is to connect two scales of cardiac contraction and study the correlation of microscopic and macroscopic variables inside a complete CVS model. This implies the computation of many heart beats in a row, which is the reason we choose a simple spherical ventricular model. Our approach in building this ventricular model was similar to Shim et al. [4] but with four major differences: we carefully chose the volume $V_{w r}$ from Fig. 4 so that the sarcomere length ranges between extreme physiological values. The pressure-force relationship derived in equation (7) was also different from the one used by Shim et al. [4]. We also used the last version from the mechanical model of Negroni and Lascano [18]. A similar 

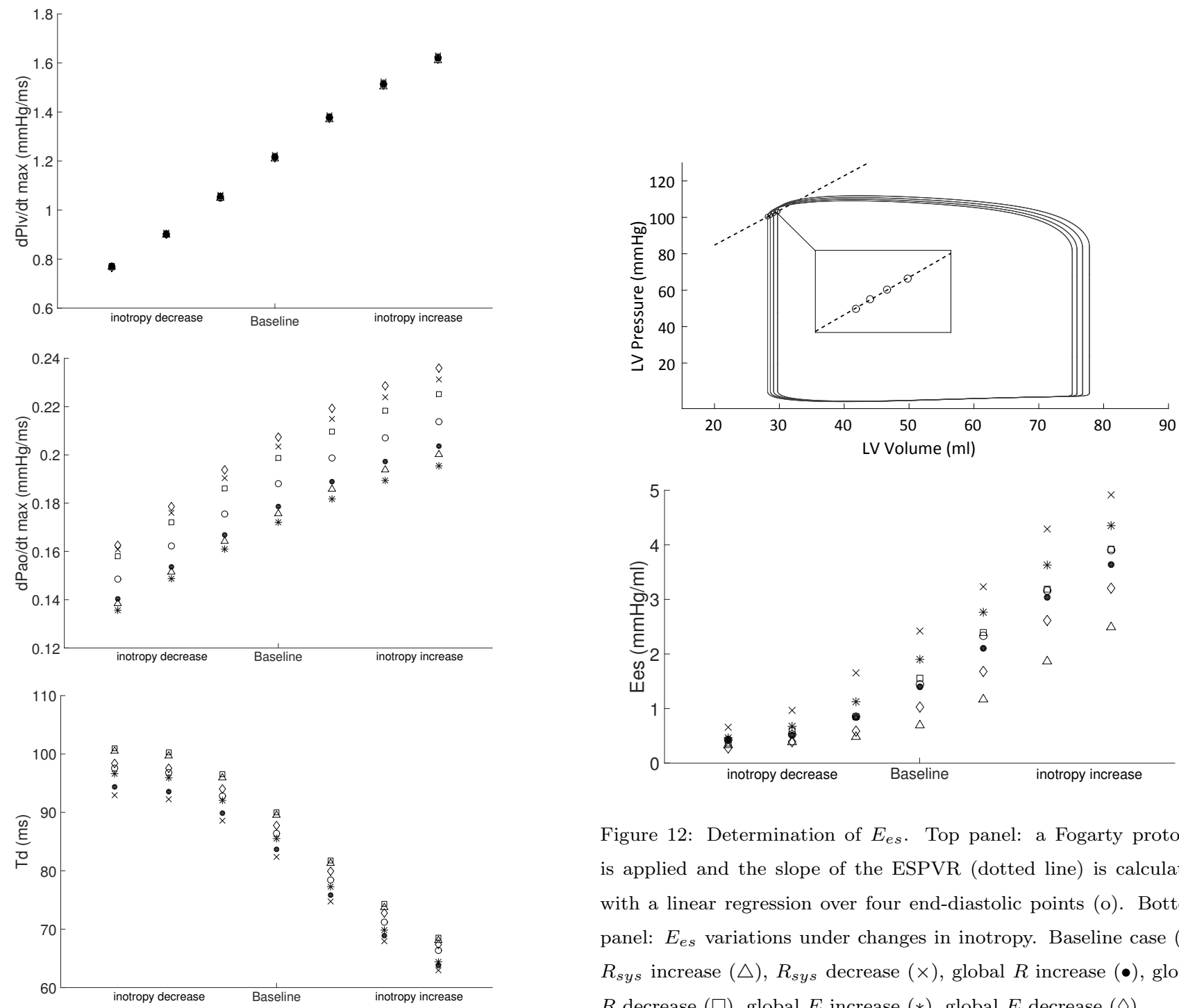

Figure 12: Determination of $E_{e s}$. Top panel: a Fogarty protocol is applied and the slope of the ESPVR (dotted line) is calculated with a linear regression over four end-diastolic points (o). Bottom panel: $E_{e s}$ variations under changes in inotropy. Baseline case (o), $R_{\text {sys }}$ increase $(\triangle), R_{\text {sys }}$ decrease $(\times)$, global $R$ increase $(\bullet)$, global $R$ decrease $(\square)$, global $E$ increase $(*)$, global $E$ decrease $(\diamond)$.

Figure 11: Cardiac contractility indices variations under changes in inotropy. Baseline case (o), $R_{\text {sys }}$ increase $(\triangle), R_{\text {sys }}$ decrease $(\times)$, global $R$ increase $(\bullet)$, global $R$ decrease $(\square)$, global $E$ increase $(*)$, global $E$ decrease $(\diamond)$. 
multiscale model was also built by Pironet et al. [5] with the first model of Negroni and Lascano [16] but not with a complete cellular description: a calcium driver was used as an input for the mechanical model.

Our results correlate well with general trends. Our multiscale model can account for a healthy behavior as shown in Fig. 5 and 6. More importantly, it is also able to reproduce pathological behaviors that originate at the cellular scale, like heart failure, and the consequences on the whole CVS. It is also able to reproduce basic hemodynamic experiments like preload variations. A theoretical study of the load-dependence of four cardiac contractility indices was also performed. We show that all these quantities are monotonic functions of inotropy. However only $\left(\mathrm{dP}_{\mathrm{LV}} / \mathrm{dt}\right)_{\max }$ can be considered as a true index of contractility since it is actually load-independent. The loaddependency of $T_{d}$, and to a lesser extent, of $\left(\mathrm{dP}_{\mathrm{AO}} / \mathrm{dt}\right)_{\max }$ remains rather small and these two measures are thus reasonably good indices of cardiac contractility. On the contrary, the load-dependency of the end-systolic elastance is large (see also [5]) and even if $E_{e s}$ increases with inotropy, it cannot be considered as an absolute index of contractility. Moreover due to this load-dependency (also observed in experiments [9]), the use of traditional timevarying elastance models to describe myocardial contraction becomes of course quite questionable.

\section{Acknowledgements}

Th. Desaive and A. Pironet are cordially acknowledged for comments and advice, especially in the identification of the hemodynamic parameters.

\section{Appendix A. Adjusted parameters}

Table A.1: Adjusted parameters

\begin{tabular}{|c|c|}
\hline Parameter & Value \\
\hline$K_{e}($ In passive force $)$ & $3.1510^{4} \mathrm{mNmm}^{-2} \mu \mathrm{m}^{-5}$ \\
\hline$L_{e}($ In passive force $)$ & $3.00 \mathrm{mNmm}^{-2} \mu \mathrm{m}^{-1}$ \\
\hline$\alpha($ In series elastic element) & $0.15 \mathrm{mNmm}^{-2}$ \\
\hline$B_{p}($ In sliding velocity $)$ & $1.75 \mathrm{~ms}^{-1}$ \\
\hline$B_{w}($ In sliding velocity $)$ & $1.225 \mathrm{~ms}^{-1}$ \\
\hline$S B V$ (Stressed Blood Volume) & $1.1610^{3} \mathrm{ml}$ \\
\hline$R_{\text {sys }}$ (Systemic resistance) & $1.3610^{3} \mathrm{mmHg} \mathrm{ms} \mathrm{ml}^{-1}$ \\
\hline$R_{p u l}$ (Pulmonary resistance) & $70.1 \mathrm{mmHg} \mathrm{ms} \mathrm{ml}^{-1}$ \\
\hline$R_{m t}$ (Mitral valve resistance) & $22.1 \mathrm{mmHg} \mathrm{ms} \mathrm{ml}^{-1}$ \\
\hline$R_{t c}$ (Tricuspid valve resistance) & $11.6 \mathrm{mmHg} \mathrm{ms} \mathrm{ml}^{-1}$ \\
\hline$R_{a v}$ (Aortic valve resistance) & $48.0 \mathrm{mmHg} \mathrm{ms} \mathrm{ml}^{-1}$ \\
\hline$R_{p v}($ Pulmonary valve resistance) & $3.51 \mathrm{mmHg} \mathrm{ms} \mathrm{ml}^{-1}$ \\
\hline$E_{a o}($ Aorta elastance $)$ & $0.615 \mathrm{mmHg} \mathrm{ml}^{-1}$ \\
\hline$E_{v c}($ Vena cava elastance $)$ & $0.0125 \mathrm{mmHg} \mathrm{ml}^{-1}$ \\
\hline$E_{p a}($ Pulmonary artery elastance) & $0.331 \mathrm{mmHg} \mathrm{ml}^{-1}$ \\
\hline$E_{p v}($ Pulmonary vein elastance $)$ & $0.0411 \mathrm{mmHg} \mathrm{ml}^{-1}$ \\
\hline$V_{l v w}($ Left ventricular wall volume) & $203 \mathrm{ml}$ \\
\hline$V_{\text {rvw }}$ (Right ventricular wall volume) & $31.3 \mathrm{ml}$ \\
\hline
\end{tabular}

\section{References}

[1] H. Suga and K. Sagawa, "Instantaneous Pressure-Volume Relationships and Their Ratio in the Excised, Supported Canine Left Ventricle," Circ. Res., vol. 35, pp. 117-126, jul 1974.

[2] D. Burkhoff and J. V. Tyberg, "Why does pulmonary venous pressure rise after onset of LV dysfunction: a theoretical analysis," Am J Physiol Hear. Circ Physiol, vol. 265, pp. H18191828, nov 1993.

[3] B. W. Smith, J. G. Chase, R. I. Nokes, G. M. Shaw, and G. Wake, "Minimal haemodynamic system model including ventricular interaction and valve dynamics," Med Eng Phys, vol. 26, no. 2, pp. 131-139, 2004.

[4] E. B. Shim, A. Amano, T. Takahata, T. Shimayoshi, and A. Noma, "The cross-bridge dynamics during ventricular contraction predicted by coupling the cardiac cell model with a circulation model," J Physiol Sci, vol. 57, no. 5, pp. 275-285, 2007. 
[5] A. Pironet, T. Desaive, S. Kosta, A. Lucas, S. Paeme, A. Collet, C. G. Pretty, P. Kolh, and P. C. Dauby, "A multi-scale cardiovascular system model can account for the load-dependence of the end-systolic pressure-volume relationship.," Biomed. Eng. Online, vol. 12, p. 8, jan 2013.

[6] S. Kosta, J. Negroni, E. Lascano, and P. Dauby, "Cell-based description of ventricular contraction in a model of the human cardiovascular system," IFAC-PapersOnLine, vol. 48, no. 20, pp. 147-152, 2015.

[7] D. Burkhoff, S. Sugiura, D. T. Yue, and K. Sagawa, "Contractility-dependent curvilinearity of end-systolic pressurevolume relations.," Am. J. Physiol., vol. 252, pp. H1218-27, jun 1987.

[8] D. A. Kass, R. Beyar, E. Lankford, M. Heard, W. L. Maughan, and K. Sagawa, "Influence of contractile state on curvilinearity of in situ end- systolic pressure-volume relations," Circulation, vol. 79, pp. 167-178, jan 1989.

[9] D. Burkhoff, P. P. de Tombe, W. C. Hunter, and D. A. Kass, "Contractile strength and mechanical efficiency of left ventricle are enhanced by physiological afterload.," Am. J. Physiol., vol. 260, pp. H569-78, feb 1991.

[10] S. R. Vaartjes and H. B. Boom, "Left ventricular internal resistance and unloaded ejection flow assessed from pressure-flow relations: a flow-clamp study on isolated rabbit hearts," Circ. Res., vol. 60, no. 5, pp. 727-737, 1987.

[11] S. G. Shroff, J. S. Janicki, and K. T. Weber, "Evidence and quantitation of left ventricular systolic resistance," Am J Physiol Hear. Circ Physiol, vol. 249, pp. H358-370, aug 1985.

[12] S. M. Davidson, D. O. Kannangara, C. G. Pretty, S. Kamoi, A. Pironet, T. Desaive, and J. G. Chase, "Modelling of the nonlinear end-systolic pressure-volume relation and volume-atzero-pressure in porcine experiments.," Conf. Proc. ... Annu. Int. Conf. IEEE Eng. Med. Biol. Soc. IEEE Eng. Med. Biol. Soc. Annu. Conf., vol. 2015, pp. 6544-7, aug 2015.

[13] T. Arts, T. Delhaas, P. Bovendeerd, X. Verbeek, and F. W. Prinzen, "Adaptation to mechanical load determines shape and properties of heart and circulation: the CircAdapt model.," Am. J. Physiol. Heart Circ. Physiol., vol. 288, pp. H1943-54, apr 2005.

[14] J. L. Puglisi, F. Wang, and D. M. Bers, "Modeling the isolated cardiac myocyte.," Prog. Biophys. Mol. Biol., vol. 85, pp. 16378 , jan 2004.

[15] K. H. W. J. ten Tusscher and A. V. Panfilov, "Alternans and spiral breakup in a human ventricular tissue model.," Am. J. Physiol. Heart Circ. Physiol., vol. 291, pp. H1088-100, sep 2006.

[16] J. A. Negroni and E. C. Lascano, "A cardiac muscle model relating sarcomere dynamics to calcium kinetics.," J. Mol. Cell. Cardiol., vol. 28, pp. 915-29, may 1996.
[17] J. A. Negroni and E. C. Lascano, "Simulation of steady state and transient cardiac muscle response experiments with a Huxley-based contraction model," J Mol Cell Cardiol, vol. 45, no. 2, pp. 300-312, 2008.

[18] J. A. Negroni, S. Morotti, E. C. Lascano, A. V. Gomes, E. Grandi, J. L. Puglisi, and D. M. Bers, " $\beta$-adrenergic effects on cardiac myofilaments and contraction in an integrated rabbit ventricular myocyte model.," J. Mol. Cell. Cardiol., vol. 81, pp. 162-75, apr 2015.

[19] E. C. Lascano, M. Said, L. Vittone, A. Mattiazzi, C. MundiñaWeilenmann, and J. A. Negroni, "Role of CaMKII in post acidosis arrhythmias: a simulation study using a human myocyte model.," J. Mol. Cell. Cardiol., vol. 60, pp. 172-83, jul 2013.

[20] Supplementary material (S1).

[21] E. K. Rodriguez, W. C. Hunter, M. J. Royce, M. K. Leppo, A. S. Douglas, and H. F. Weisman, "A method to reconstruct myocardial sarcomere lengths and orientations at transmural sites in beating canine hearts," Am J Physiol Hear. Circ Physiol, vol. 263, pp. H293-306, jul 1992.

[22] J. A. Negroni and E. C. Lascano, "Concentration and Elongation of Attached Cross-bridges as Pressure Determinants in a Ventricular Model," J. Mol. Cell. Cardiol., vol. 31, pp. 15091526, aug 1999.

[23] Edwards Lifesciences, Normal Hemodynamic Parameters and Laboratory Values. Available at http: //ht.edwards.com/scin/edwards/sitecollectionimages/ edwards/products/presep/ar04313hemodynpocketcard.pdf. Accessed 2016-05-22.

[24] LiDCO, Normal hemodynamic parameters. Available at http: //www.lidco.com/clinical/hemodynamic.php. Accessed 201605-22.

[25] A. Pironet, T. Desaive, J. Geoffrey Chase, P. Morimont, and P. C. Dauby, "Model-based computation of total stressed blood volume from a preload reduction manoeuvre.," Math. Biosci., vol. 265, pp. 28-39, jul 2015.

[26] T. Arts, P. H. Bovendeerd, F. W. Prinzen, and R. S. Reneman, "Relation between left ventricular cavity pressure and volume and systolic fiber stress and strain in the wall.," Biophys. J., vol. 59, pp. 93-102, jan 1991.

[27] P. Bovendeerd, T. Arts, J. Huyghe, D. van Campen, and R. Reneman, "Dependence of local left ventricular wall mechanics on myocardial fiber orientation: A model study," $J$. Biomech., vol. 25, pp. 1129-1140, oct 1992.

[28] D. J. Beuckelmann, M. Nabauer, and E. Erdmann, "Intracellular calcium handling in isolated ventricular myocytes from patients with terminal heart failure," Circulation, vol. 85, pp. 1046-1055, mar 1992.

[29] D. J. Beuckelmann, M. Nabauer, and E. Erdmann, "Alterations 
of $\mathrm{K}+$ currents in isolated human ventricular myocytes from patients with terminal heart failure," Circ. Res., vol. 73, pp. 379385, aug 1993.

[30] Y. Wang and J. A. Hill, "Electrophysiological remodeling in heart failure.," J. Mol. Cell. Cardiol., vol. 48, pp. 619-32, apr 2010.

[31] R. E. Klabunde, Cardiovascular Physiology Concepts. 2004.

[32] D. A. Kass, W. L. Maughan, Z. M. Guo, A. Kono, K. Sunagawa, and K. Sagawa, "Comparative influence of load versus inotropic states on indexes of ventricular contractility: experimental and theoretical analysis based on pressure-volume relationships.," Circulation, vol. 76, pp. 1422-36, dec 1987.

[33] D. Adler, E. Scott Monrad, E. H. Sonnenblick, O. M. Hess, and H. P. Krayenbuehl, "Time to dp/dt max , a useful index for evaluation of contractility in the catheterization laboratory," Clin. Cardiol., vol. 19, pp. 397-403, may 1996.

[34] P. Morimont, B. Lambermont, T. Desaive, N. Janssen, G. Chase, and V. D'Orio, "Arterial dP/dtmax accurately reflects left ventricular contractility during shock when adequate vascular filling is achieved.," BMC Cardiovasc. Disord., vol. 12, p. 13 , jan 2012 . 